\title{
Effects of Flying Height and Compositions of Antenna and Media on Near-Field Optics for Thermally Assisted Magnetic Recording
}

\author{
S. Kudoh, Y. Moriyama*, J. Kim*, K. Nakagawa*, and A. Itoh* \\ Graduate Course of Electronic Engineering, Graduate school of Science \& Technology, Nihon University, \\ 7-24-1 Narashinodai, Funabashi, Chiba 274-8501, Japan \\ *Department of Electronics \& Computer Science, College of Science \& Technology, Nihon University, \\ 7-24-1 Narashinodai, Funabashi, Chiba 274-8501, Japan \\ **Department of Electrical \& Electronic Engineering, Toyohashi University of Technology, \\ 1-1 Tempaku, Toyohashi, Aichi 441-8580, Japan
}

Thermally assisted magnetic recording (TAMR) is a promising technology to achieve a recording density of over several Tbit/inch ${ }^{2}$. We analyzed the use of a plasmon antenna as well as the effect of a particle medium with Finite-Difference Time-Domain (FDTD) method. Using a plasmon antenna, we analyzed the dependence of flying height $(\mathrm{FH})$ on the power intensity $\left(E^{2}\right)$. FH is the distance between the medium and the antenna. We also analyzed the dependence of full width half maximum (FWHM) on near-field optics. We investigated the influence of different compositions of the antenna and of the media on plasmon resonance. Three compositions of the particle medium were $\mathrm{Au}, \mathrm{Pt}$, and Co. For metallic particles, the FWHM was less than $15 \mathrm{~nm}$ when FH was $5 \mathrm{~nm}$ to $20 \mathrm{~nm}$. The peak intensity for the particle medium was higher than that of the continuous film across various values for $\mathrm{FH}$. Furthermore, the overall power distribution was higher as well. These results show that particles positioned at the apex of the antenna can be heated by a small, concentrated spot.

Key words: thermally assisted magnetic recording, TAMR, near-field optics, flying height, particle media, FDTD

\section{Introduction}

Recently, one of the goals of the recording research field is to achieve a recording density of over several Tbit/inch ${ }^{2}$. To reach this recording density, extremely small magnetic grains are needed, specifically, 1 bit in an area measuring less than $25 \times 25 \mathrm{~nm}^{2}$. Due to the physical limitations of the magnetization of such small grains, magnetic stability is easily affected by thermal energy. Granular media and particle media, which have high uniaxial anisotropic energy $\left(K_{\mathrm{u}}\right)$, are required to overcome this problem in magnetic recording 1 ), 2). Particular attention has been given to the use of $L 1_{0}$ type $\mathrm{FePt}$ for high density media ${ }^{2), 3)}$. An extremely intense recording magnetic field is needed to record on high $K_{\text {u }}$ media. A normal optical system such as a laser cannot focus down to this size due to the diffraction limit. Thermally assisted magnetic recording (TAMR) can break this limit using optical spots to heat the medium locally by near-field optics. Optical spots less than $50 \mathrm{~nm}$ are required for a recording density over several Tbit/inch ${ }^{24}$.

To obtain high optical power by using near-field optics, the structure and composition of the antenna are important. The plasmon antenna of the head should generate a high power in a nanosize area by near-field optics. Several researches have reported various hybrid heads, such as the waveguide type hybrid head ${ }^{5}$, the Surface plasmon and Magnetic field applicable Synchronously Hybridized (SMASH) head ${ }^{6)}$, and a beaked triangle antenna ${ }^{7}$. In addition, it is reported that the resonance wavelength depends on the shape of the plasmon antenna and its compositions ${ }^{8), 9)}$.

There are a few additional points to consider when using TAMR. First, the distance from the antenna affects the peak intensity and its full width half maximum (FWHM). We call the distance from the plasmon antenna to the medium flying height (FH). In general, with increasing $\mathrm{FH}$ for the continuous film, the peak intensity decreases and its FWHM broadens ${ }^{10)}, 11$. Second, the length of the plasmon antenna and its composition especially influence plasmon resonance wavelength and optical efficiency ${ }^{8), 9}$. Third, there is a dependence of both the intensity and FWHM on the optical spot. In our previous study, we studied TAMR using a plasmon antenna with a particle medium ${ }^{12)}$. We decreased the optical spot to less than $15 \mathrm{~nm}$ and increased the optical intensity to over twice that which can be obtained from the use of conventional continuous film. The influences of $\mathrm{FH}$ and plasmon resonance on the peak intensity and its FWHM for the particle medium have not been clarified. Further research is required to better understand the effects of $\mathrm{FH}$, and its influence on plasmon resonance with a particle medium.

In this paper, we studied the dependence of $\mathrm{FH}$ on near-field optics and also the effect of plasmon resonance with particle media by Finite-Difference Time-Domain (FDTD) method. The media were ordered metallic particles, $\mathrm{Au}, \mathrm{Pt}$, and Co. Effects of particle media and metallic film are compared. 


\section{Influence on Near-Field Optics by Flying Height with Particle Media}

The power intensity $\left(E^{2}\right)$ was calculated for the distribution on the media generated by the antenna, using FDTD, which has worked well for analyzing and optimizing antennas. The volume of the simulation was $1800 \times 1800 \times 250 \mathrm{~nm}^{3}$. A non-uniform cell was used in these simulations. The cell size in this simulation was the smallest at $1 \times 1 \times 1 \mathrm{~nm}^{3}$ at the center of the simulation model, near the apex of the antenna. Mur's absorption boundary condition was used in calculating the boundary conditions ${ }^{13)}$. A simulation model of the antenna was designed in order to investigate the influence of $\mathrm{FH}$ on $E^{2}$, as shown in Fig. 1.

The plasmon antenna was arranged with the longitudinal direction parallel to $\mathrm{X}$-axis in $\mathrm{X}-\mathrm{Y}$ plane. Both the antenna and particle medium compositions in this calculation were made from Pt. Antenna length $(L)$ of the longitudinal direction was $400 \mathrm{~nm}$, the apex radius $(r)$ was $25 \mathrm{~nm}$, the apex angle $(\theta)$ was $40^{\circ}$, and the thickness was $35 \mathrm{~nm}$. The $\mathrm{Pt}$ particles were spherical and arrayed in a close-packed structure. The diameter $(d)$ and the spacing $(s)$ of the particles were set to $15 \mathrm{~nm}$ and $10 \mathrm{~nm}$, respectively. The Drude model was used to determine the relative dielectric constant. The coefficients of the optical properties of each composition, such as refractive index $(n)$ and extinction coefficient $(k)$ were set to $n=2.76, k=4.84^{14)}$. Relative permeability of the antenna and particle media compositions were fixed at 1 . The FH between particle media and antenna were set at various increments from $5 \mathrm{~nm}$ to $20 \mathrm{~nm}$.

The wavelength $(\lambda)$ of incident light was $780 \mathrm{~nm}$. The optical source was set $60 \mathrm{~nm}$ from the plasmon antenna, which was a Gaussian distributed plane FDTD source. The diameter of the optical spot $\left(1 / e^{2}\right)$ was set to be equal to the wavelength of the incident light. The peak intensity of the electric field at the center of the light source was $1 \mathrm{~V} / \mathrm{m}$. The electric field of polarized

(a) Plane view

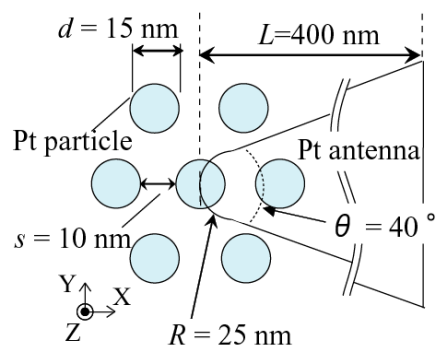

(b) Cross sectional side view

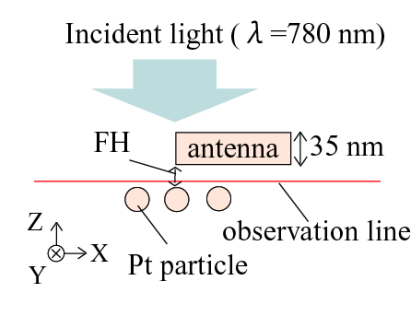

Fig. 1 Schematic diagram of (a) the simulation model for particle medium and (b) the cross section of simulation model. The Pt particles were spherical and arrayed in a close-packed structure. The diameter $(d)$ and the spacing $(s)$ of the particles were set to $15 \mathrm{~nm}$ and $10 \mathrm{~nm}$, respectively. light was aligned with the longitudinal direction of the antenna. Observation of $E^{2}$ in the Z-axis, perpendicular to the plane of the antenna, was measured at a height 1 $\mathrm{nm}$ from the medium.

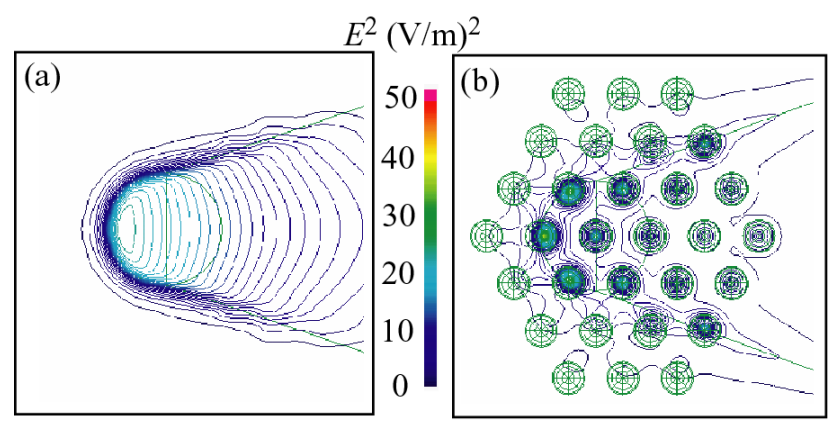

Fig. $2 E^{2}$ distribution of near-field optics in case of (a) continuous film and (b) patterned medium. The flying height was $5 \mathrm{~nm}$.

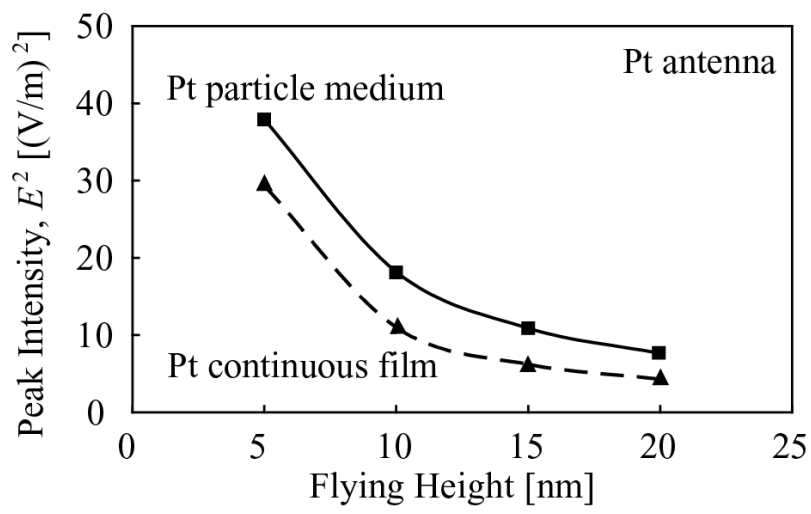

Fig. 3 Dependence of peak intensity as a function of FH. Solid line and dashed line represent the curves of the particle medium and the continuous film, respectively.

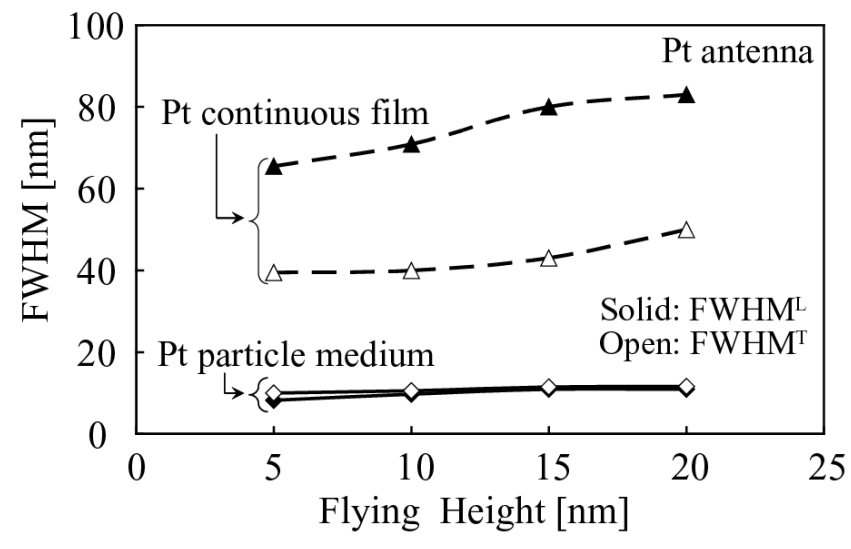

Fig. 4 Dependence of FWHM as a function of FH. Solid line and dashed line represent the curves of the particle medium and the continuous film, respectively. The open symbol and solid symbol represent the plot of the FWHM of the longitudinal (FWHM ${ }^{\mathrm{L}}$ ) and transverse $\left(\mathrm{FWHM}^{\mathrm{T}}\right)$ directions of the antenna, respectively. 
The distribution of the $E^{2}$ was highly concentrated above the particle under the apex of the antenna with a particle medium. Figure 2 shows $E^{2}$ distribution of $\mathrm{FH}=$ $5 \mathrm{~nm}$ of particles medium and continuous film. For continuous film, the $E^{2}$ distribution broadened around antenna apex, however for particle medium, distribution concentrated around a narrow area by the particles. Figure 3 shows $E^{2}$ values as a function of $\mathrm{FH}$ with a particle medium and a continuous film. The $E^{2}$ increased with decreasing $\mathrm{FH}$ for both cases. The $E^{2}$ of the particle medium was higher than that of the continuous film across all $\mathrm{FH}$. When $\mathrm{FH}=5 \mathrm{~nm}, E^{2}$ was about $40(\mathrm{~V} / \mathrm{m})^{2}$ with the particle medium. The reason for the enhancement in the peak intensity relative to the particle medium could be expected by the interaction between the antenna and particles. This effect was more obvious in FWHM. Figure 4 shows the FWHM of the special intensity distribution as a function of $\mathrm{FH}$ for both media. For the continuous film, the FWHMs of the longitudinal (FWHM ${ }^{\mathrm{L}}$ ) and transverse $\left(\mathrm{FWHM}^{\mathrm{T}}\right)$ directions of the antenna were over $60 \mathrm{~nm}$ and over $40 \mathrm{~nm}$ at $\mathrm{FH}=5 \mathrm{~nm}$, respectively. Furthermore, as FH increased the FWHMs spread out in both directions. The FWHMs for the particle medium were independent of the antenna direction with values that were less than $15 \mathrm{~nm}$ when $\mathrm{FH}$ was $5 \mathrm{~nm}$ to $20 \mathrm{~nm}$. The FWHM ${ }^{\mathrm{L}}$ concentrated to one sixth or less, and the FWHM $^{\mathrm{T}}$ concentrated to one fourth or less with the particle medium. This fact means the spatial spread of the $E^{2}$ was determined not only by the antenna length but also by the particle shape of the medium. As a result, the particle medium can concentrate the optical spot more than the continuous film. Therefore, the combination of the plasmon antenna with the particle medium proved to be an effective method for heating the local area.

\section{Particle and Antenna Composition Dependency}

It has already been reported that an optical spot can be squeezed and extremely near-field optics can be generated with a particle medium ${ }^{12}$. What has not yet been analyzed is the influence of a particle medium on plasmon resonance. Furthermore, it is necessary to confirm whether different compositions of the antenna and particle media have a similar effect on plasmon resonance. Therefore, we investigated $E^{2}$ in order to clarify the effects between the antenna and medium on the plasmon resonance.

Figure 5 shows the schematic view of the simulation model. The antennas were $\mathrm{Au}$ and $\mathrm{Pt}$ with various $L$. Three media compositions studied were Au, $\mathrm{Pt}$, and Co. The optical properties are shown in Table $1^{14), 15)}$. The spherical particles were arranged in a close-packed structure. The $d$ and $s$ were $15 \mathrm{~nm}$ and 10 $\mathrm{nm}$, respectively. The $\mathrm{FH}$ was $5 \mathrm{~nm}$. The $\lambda$ for the incident light was set at various increments from 400 to (a) Plane view

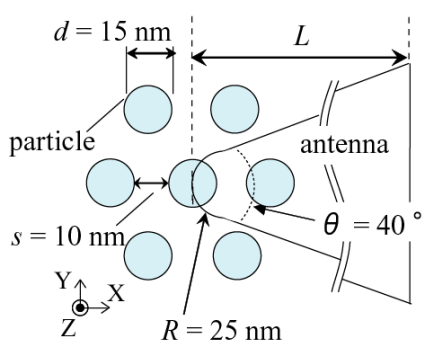

(b) Cross sectional side view

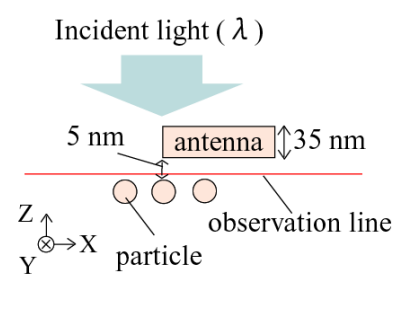

Fig. 5 The schematic diagram of (a) the simulation model for the plasmon antenna with the spherical metal particles and (b) its detailed illustration around the apex of the antenna.

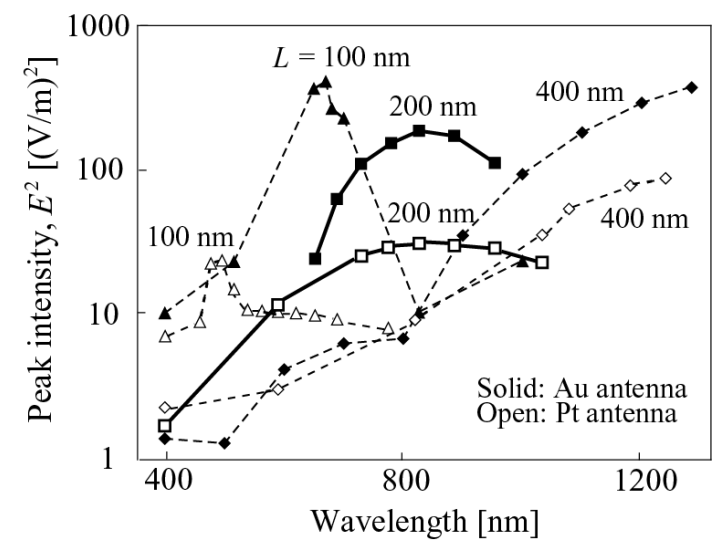

Fig. 6 Wavelength dependence of power intensities of $\mathrm{Au}$ and Pt antennas with a few antenna lengths (L). The data of $L=100$ and $400 \mathrm{~nm}$ have already been reported ${ }^{9)}$.

Table 1 Optical properties of metallic compositions ${ }^{14), 15)}$

\begin{tabular}{|c|c|c|c|c|c|c|}
\hline \multirow{2}{*}{$\begin{array}{c}\text { Wavelength } \\
\text { [nm] }\end{array}$} & \multicolumn{2}{|c|}{$\mathrm{Au}$} & \multicolumn{2}{|c|}{$\mathrm{Pt}$} & \multicolumn{2}{|c|}{ Co } \\
\hline & $\begin{array}{l}\text { Reflective } \\
\text { Index }(n)\end{array}$ & $\begin{array}{c}\text { Extinction } \\
\text { Coefficient }(k)\end{array}$ & $\begin{array}{l}\text { Reflective } \\
\text { Index }(n)\end{array}$ & $\begin{array}{c}\text { Extinction } \\
\text { Coefficient }(k)\end{array}$ & $\begin{array}{l}\text { Reflective } \\
\text { Index }(n)\end{array}$ & $\begin{array}{c}\text { Extinction } \\
\text { Coefficient }(k)\end{array}$ \\
\hline 652 & 0.166 & 3.15 & 2.38 & 4.26 & 2.25 & 4.27 \\
\hline 688 & 0.16 & 3.8 & 2.51 & 4.43 & 2.31 & 4.45 \\
\hline 729 & 0.164 & 4.35 & 2.63 & 4.63 & 2.4 & 4.64 \\
\hline 775 & 0.174 & 4.86 & 2.76 & 4.84 & 2.53 & 4.88 \\
\hline 826 & 0.188 & 5.39 & 4.92 & 5.07 & 2.65 & 5.16 \\
\hline 886 & 0.21 & 5.88 & 3.1 & 5.32 & 2.78 & 5.5 \\
\hline 953 & 0.236 & 6.47 & 3.29 & 5.61 & & \\
\hline
\end{tabular}




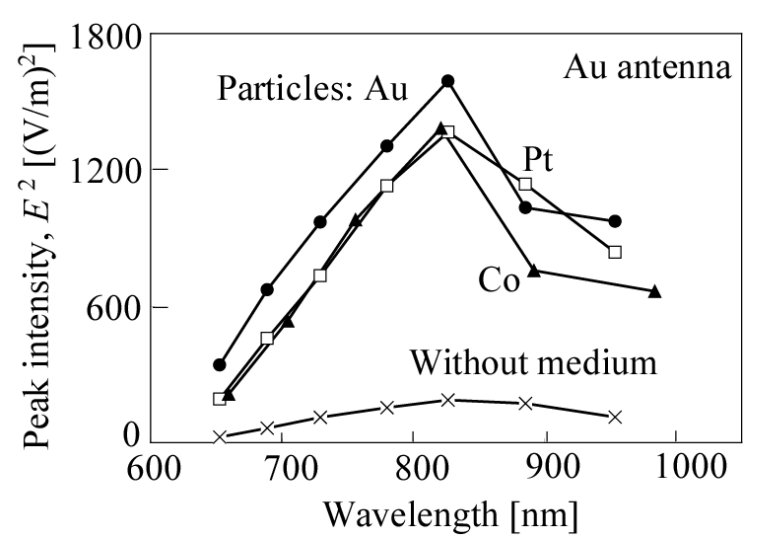

Fig. 7 Change of peak intensity with and without spherical particle media as a function of wavelength. The media were $\mathrm{Au}, \mathrm{Pt}$, and $\mathrm{Co}$. The antenna was $\mathrm{Au}$ at $L=200 \mathrm{~nm}$.

$1300 \mathrm{~nm}$. The distance between the optical source and the plasmon antenna was $60 \mathrm{~nm}$. The other simulation conditions were the as same as described in section 2 .

Figure 6 shows the $E^{2}$ as a function of $\lambda$ in case of $\mathrm{Au}$ and $\mathrm{Pt}$ antennas with various $L$ without medium. We reported before that the $E^{2}$ values were shown to be a function of $\lambda$ and the $L^{9)}$. When the $L=400 \mathrm{~nm}$, the $E^{2}$ was low at around $\lambda=780 \mathrm{~nm}$, which was the wavelength we used ${ }^{10)}$. Therefore, the wavelength dependency was examined in addition to determine the optimal $L$ and antenna composition. High $E^{2}$ at around $\lambda=780 \mathrm{~nm}$ was obtained for the Au antenna with $L=$ $200 \mathrm{~nm}$. This fact means the plasmon resonance was changed by $L$. The optimal antenna conditions at around $\lambda=780 \mathrm{~nm}$ were Au with $L=200 \mathrm{~nm}$.

In Fig. 7 , the $E^{2}$ of various media compositions are shown as a function of $\lambda$ using the Au antenna with $L=$ $200 \mathrm{~nm}$. In all media, the $E^{2}$ values rose up rapidly with an increase of $\lambda$ from 650 to $820 \mathrm{~nm}$, and suddenly dropped with a further increase of $\lambda$. The change of $E^{2}$ without media was much smaller than that of any other particle media. The maximum $E^{2}$ value was obtained with an Au antenna and was about 9 times higher than that without media. The maximum $E^{2}$ for each medium was $\mathrm{Au}=1600(\mathrm{~V} / \mathrm{m})^{2}, \mathrm{Pt}=1365(\mathrm{~V} / \mathrm{m})^{2}, \mathrm{Co}=1380$ $(\mathrm{V} / \mathrm{m})^{2}$. This means that the output of the near-field optics can be further concentrated by a particle medium.

In addition, in all medium compositions, both the FWHM $^{\mathrm{L}}$ and the $\mathrm{FWHM}^{\mathrm{T}}$ were less than $10 \mathrm{~nm}$ across various $\lambda$. Therefore, it was found that we could effectively concentrate the optical spot with a particle medium. When using the particle medium, high $E^{2}$ and narrow FWHM can be achieved simultaneously by the plasmon resonance. This plasmon resonance is effective for TAMR.

\section{Conclusion}

The influences of $\mathrm{FH}$ and composition of the antenna and medium on plasmon resonance of the near-field optics were investigated by FDTD method. With particle medium, $E^{2}$ was higher than continuous film across various $\mathrm{FH}$. The dependence of $\mathrm{FH}$ on FWHM was improved by a particle medium. In plasmon resonance with particle media, the $\mathrm{Au}$ particle medium showed the highest $E^{2}$ value over other compositions. However, when using other compositions, the $E^{2}$ and FWHM showed a high power with extreme concentration similar to when same composition of the antenna and medium. Particle media and plasmon resonance are very effective for concentrating the optical spot for thermally assisted magnetic recording.

Acknowledgements This work is partially supported by a Grant-in-Aid for Scientific Research of the Ministry of Education, Culture, Sports, Science and Technology in Japan No. 18560348.

\section{References}

1) J. J. M. Ruigrok, R. Coehoorn, S. R. Cumpson and H. W. Kesteren: J. Appl. Phys. 87, 5398 (2000).

2) D. Weller, A. Moser, L. Folks, M. E. Best, W. Lee, M. F. Toney, M. Schwickert, J-U. Thiele and M. F. Dorener: IEEE Trans. Magn., 36, 10 (2000).

3) J. M. Qiu, J. H. Judy, D. Weller and J. P. Wang: J. Appl. Phys. 97, 10J319 (2005).

4) A.V. Itagi, D. D. Stancil, J. A. Bain and T. E. Schlesinger: Appl. Phys. Lett. 83, 4474 (2003).

5) T. E. Schlesinger, T. Rausch, A. Itagi, J. Zhu, J. A. Bain, and D. D. Stancil: J. Appl. Phys, 41, 1821 (2002).

6) S. Miyanishi, N. Iketani, K. Takayama, K. Innami, I. Suzuki, T. Kitazawa, Y. Ogimoto, Y. Murakami, K. Kojima, and A. Takahashi: IEEE Trans. Magn., 41, 2817 (2005).

7) T. Matsumoto, Y. Anzai, T. Shintani, K. Nakamura, and T. Nishida: Opt. Lett., 31, 259 (2006).

8) T. Matsumoto, T. Shimano, H. Saga, and H. Sukeda: J. Appl. Phys., 95, 3901 (2004).

9) S. Kudoh, J. Kim, K. Nakagawa, and A. Itoh: J. Magn. Soc. Jpn., 30, 612 (2006).

10) N. Mori, J. Kim, K. Nakagawa, and A. Itoh: J. Magn. Soc. Jpn., 30, 604 (2006).

11) K. Nakagawa, J. Kim, and A. Itoh: J. Appl. Phys., 99, 08F902 (2006).

12) K. Nakagawa, J. Kim, and A. Itoh: J. Appl. Phys., 101, 09H504 (2007).

13) Gerrit Mur: IEEE Trans. Electromagn. Compat., EMC-23, 377 (1981).

14) Handbook of Optical Constants of Solids I, Edword D. palik ed., (Academic Press, San Diego, 1998), p. 286, p. 333.

15) Handbook of Optical Constants of Solids II, Edword D. palik ed., (Academic Press, San Diego, 1998), p. 435.

Received Sep. 07, 2007; Revised Oct. 19, 2007;

Accepted Nov. 13, 2007 